Proceedings of the 6th Polish Symposium of Physics in Economy and Social Sciences (FENS2012), Gdańsk, Poland

\title{
Rayleigh's Distribution, Wigner's Surmise and Equation of the Diffusion
}

\author{
R. WOJNAR \\ IPPT PAN, A. Pawińskiego 5b, 02-106 Warsaw, Poland
}

\begin{abstract}
After summaries on Rayleigh's distribution and Wigner's surmise, the time evolution of Rayleigh-Wigner's statistics is studied and a suitable diffusion type equation is proposed. Also the variance and kurtosis of time evolution of Rayleigh's distribution are calculated. Obtained results may be useful in description of physical, social and biological processes.
\end{abstract}

DOI: 10.12693 /APhysPolA.123.624

PACS: 05.40.Jc, 05.70.Ln, 82.39.Rt, 87.85.gj, 89.65.Gh, 89.65.Lm, 21.10.-k

\section{Introduction}

Non-Gaussian distributions occur in systems that do not follow strictly the prescriptions of standard statistics.

Important example of non-Gaussian statistics is distribution which was introduced by Lord Rayleigh in connection with the problem of interference of harmonic oscillations with random phases $[1,2]$. This distribution is known also as Wigner's surmise distribution giving a remarkably good description of the level repulsion observed in neutron scattering [3, 4]. As the mathematical form of both is identical, we call them Rayleigh-Wigner's distribution (RWD).

Rayleigh-Wigner's distribution appears in computation of the large zeros of Riemann's zeta function on the critical line, which according to the MontgomeryOdlyzko law have the same statistical properties as the distribution of eigenvalue spacings in a Gaussian unitary ensemble [5-9]. Rayleigh-Wigner's distribution is also met in social sciences; e.g. the bus system in Cuernavaca, Mexico, is subject to this distribution [10]. This wide range of applications of RWD is a new illustration of Wigner's opinion on unreasonable effectiveness of mathematics [11].

\subsection{Rayleigh's distribution}

As it is well known, superposition of orthogonal phase shifted sinusoid oscillations leads to complex harmonic motions, known as Lissajous' curves. In particular, the resultant of two isoperiodic vibrations of equal amplitude is wholly dependent upon their phase relation. If such vibrations are colinear and their phases differ by half a period, the resultant vanishes.

John William Strutt (Baron Rayleigh, known also as Lord Rayleigh) inquired in 1880 what results from the composition of a large number $n$ of equal vibrations of amplitude unity, of the same period, and of phases accidentally determined.

The intensity of the resultant, represented by the square of the amplitude, depends of course, upon the manner in which the phases are distributed, and may vary from zero to $n^{2}$. A question arises whether it exists a definite intensity which becomes more and more probable when $n$ is increased without limit.

\subsubsection{Two opposite phases only}

First, there is analysed the case in which the possible phases are restricted to two opposite phases, which is equivalent to regarding the amplitudes as at random positive or negative. If there be as many positive as negative, the result is zero, if, on the other hand, all signs be the same, the intensity is $n^{2}$.

Adopting the Bernoulli binomial distribution method, Rayleigh considered $N$ independent combinations, each consisting of $n$ unit vibrations. When $N$ is sufficiently large, the statistics become regular and the number of combinations in which the resultant amplitude is found to be $x$ may be denoted by $N f(n, x)$, where $f$ is definite function of $n$ and $x$.

Let each of the $N$ combinations receives another random contribution of \pm 1 . Only those combinations can subsequently possess a resultant $x$ which originally had amplitudes $x-1$ and $x+1$. Half of the former, and half of the latter number will acquire the amplitude $x$, so that the number required is

$$
\frac{1}{2} N f(n, x-1)+\frac{1}{2} N f(n, x+1) .
$$

Because this should be identical with the number corresponding to $n+1$ and $x$, we obtain

$$
f(n+1, x)=\frac{1}{2} f(n, x-1)+\frac{1}{2} f(n, x+1),
$$

which can be written in the form

$$
\begin{aligned}
& f(n+1, x)-f(n, x) \\
& \quad=\frac{1}{2}[f(n, x-1)-2 f(n, x)+f(n, x+1)] .
\end{aligned}
$$

This can be regarded as a difference counterpart of the differential heat (or diffusion) equation [12],

$$
\frac{\partial f}{\partial n}=\frac{1}{2} \frac{\partial^{2} f}{\partial x^{2}}
$$

Its special solution reads 


$$
f(n, x)=\frac{A}{\sqrt{n}} \mathrm{e}^{-x^{2} / n},
$$

in which $A$ is a normalizing constant, $A=1 / \sqrt{2 \pi}$. It is, of course, the Gaussian distribution.

\subsubsection{Phases distributed at random}

Next, it is considered a problem in which the phases of the $n$ unit vibrations are distributed at random over the entire period. The resultant amplitude is denoted by $r$ and the phase by $\theta$, and thus any point $(x, y)$ represents the vibration of amplitude $r$ and phase $\theta$. Of the large number $N$ of points

$$
N f(n, x, y) \mathrm{d} x \mathrm{~d} y
$$

are to be found within the infinitesimal area $\mathrm{d} x, \mathrm{~d} y$ around the point $(x, y)$.

Now, it is supposed that $n / 2$ vibrations is distributed at random along the $x$-axis, and $n / 2$ along $y$, and let us inquire into the probabilities of the various resultants. The probability that the end of the representative line, in other words the representative point, lies in the rectangle $\mathrm{d} x \mathrm{~d} y$ around the point $(x, y)$ is, according with the previous expression,

$$
f(n, x, y) \mathrm{d} x \mathrm{~d} y=\frac{1}{\pi n} \mathrm{e}^{-\frac{x^{2}+y^{2}}{n}} \mathrm{~d} x \mathrm{~d} y .
$$

Substituting polar coordinates $r, \theta$, the rectangular coordinates are

$$
x=r \cos \theta, \quad y=r \sin \theta .
$$

Integrating with respect to $\theta$, it is seen that the probability of the representative point of the resultant lying between the circles $r$ and $r+\mathrm{d} r$ is

$$
f_{\mathrm{R}}(n, r) \mathrm{d} r=\frac{2}{n} \mathrm{e}^{-r^{2} / n} r \mathrm{~d} r .
$$

If the amplitude of each component be $\alpha$, instead of unity, the probability of a resultant amplitude between $r$ and $r+\mathrm{d} r$ is

$$
f_{\mathrm{R}}(n, r ; \alpha) \mathrm{d} r=\frac{2}{n \alpha^{2}} \mathrm{e}^{-r^{2} / n \alpha^{2}} r \mathrm{~d} r .
$$

The result is a function of $n$ and $\alpha$ only through $n \alpha^{2}$. Hence, the standard form of Rayleigh's probability density function is

$$
f_{\mathrm{R}}(x ; a)=\frac{x}{a^{2}} \mathrm{e}^{-x^{2} / 2 a^{2}}
$$

for $x>0$ and parameter $a>0$. In probability theory and statistics, the Rayleigh distribution is an example of continuous probability distribution.

\subsection{Wigner's surmise}

Statistics of energy spacings is the important problem raised by the appearance of the nuclear spectra at high energies, such as that of uranium $\mathrm{U}^{239}$, cf. [4, 13]. The specific problem concerns the probability of succession of spacings $s_{1}, s_{2}, \ldots, s_{n}$ between adjacent energy levels. Wigner when asked, in the course of a meeting - to guess the form of the density, proposed ad hoc the form of a Gaussian multiplied by $s$.
Let $c$ be the probability of an energetic level in the unit energy interval. If the values of the levels were independent, the probability $P$ that there is no levels within the distance $h$ from a given level would obey the equation

$$
P(s+h)=P(s)-c P(s) h
$$

for very small $h$. This leads to the difference equation

$$
\frac{P(s+h)-P(s)}{h}=-c P(s)
$$

and for $h \rightarrow 0$ gives

$$
\frac{\mathrm{d} P}{\mathrm{~d} s}=-c P
$$

and

$$
P(s)=\mathrm{e}^{-c s},
$$

which means that the exponential law would follow.

Actually, however, the probability of a level right next to another one is proportional to the distance therefrom. This suggest that

$$
P(s+h)=P(s)-c P(s) h s
$$

or

$$
\frac{\mathrm{d} P}{\mathrm{~d} s}=-c P s
$$

Hence

$$
P(s)=C \mathrm{e}^{-c s^{2} / 2},
$$

where $C$ is a positive constant. From this the WSD can be obtained by differentiation

$$
f_{\mathrm{W}}(s)=-\frac{\mathrm{d} P}{\mathrm{~d} s}=C s \mathrm{e}^{-c s^{2} / 2} .
$$

Wigner was not completely sure that his guess is correct. He was therefore surprised when some years later, Mehta [14] and Gaudin [15] found that the Wigner surmise is usable for practical comparisons.

For the independent positive variable $x$, and the positive constant $a$ the mathematical form of Wigner surmise distribution is

$$
f_{\mathrm{W}}(x)=2 a x \mathrm{e}^{-a x^{2}} .
$$

The position of maximum and the maximal value are given by

$$
x_{\max }=\frac{1}{\sqrt{2 a}} \text { and } f_{\mathrm{W}}\left(x_{\max }\right)=\sqrt{2 a} \mathrm{e}^{-1 / 2} .
$$

The mathematical form of the Wigner surmise distribution $f_{\mathrm{W}}(x)$ is identical with Rayleigh's distribution $f_{\mathrm{R}}(x)$. Notice, however, that the Wigner distribution was derived for the one-dimensional energy levels problem, while Rayleigh discussed system of two-dimensions.

\subsection{Another examples of RWDs applications}

A Rayleigh distribution is often observed when the overall magnitude of a vector is related to its directional components.

Two-dimensional Maxwell's distribution in cylindrical coordinates has form of RWD. Similarly, the RWD is met when wind velocity is analyzed into its orthogonal 
2-dimensional vector components. Assuming that the magnitude of each component is uncorrelated, normally distributed with equal variance, and zero mean, then the overall wind speed (vector magnitude) is described by a RWD.

The RWD arises in the case of random complex numbers whose real and imaginary components are independently and identically distributed (i.i.d.) Gaussian. In that case, the absolute value of the complex number is Rayleigh-distributed.

The aforementioned problems are in obvious manner analogous to problem described in Sect. 1.1 and belonging to Lord Rayleigh.

Public transport in Mexico is different to that in Europe. First, no leading companies are responsible for city transport. There are no timetables for city buses and sometimes not even well-defined bus stops. The driver is usually the owner of the bus and so his aim is to maximize the income. Because every passenger boarding the bus has to pay, the driver tries to collect the largest possible number of passengers. When not regulated, the time interval during which two subsequent buses pass a given point will display a Poissonian distribution. This is a consequence of the absence of correlations between the motion of different buses. Such a situation is, however, not attended by the drivers because then the probability density that two buses will arrive at a bus stop within short time interval is large. In this case, the first bus collects all the waiting passengers and the second bus that arrives slightly later will find the stop practically empty. This reasoning produces certain correlations between buses, which change the Poisson process into RWD [16-18]. This process differs from the situation on financial markets, where correlations in time produce symmetric distributions [19].

Šba compared distribution of the bird-to-bird distances with the bumper-to-bumper distances between parking autos and found that the both distributions are in good agreement with RWD [20].

Rayleigh-Wigner's distribution is observed also in results of school examines, in the case when the students obtain some basic knowledge, but do not master an advanced one. Then the peak of the distribution is shifted to left $[21,22]$.

\subsection{Numerical evidence for the Wigner surmise}

The "Wigner surmise" states that the probability density function of the eigenvalue spacings of a random system follows the Rayleigh distribution. Thus far the surmise has been proved mathematically only for systems described by the "Gaussian orthogonal ensemble (GOE)" of matrices. Random matrices associated with practical engineering structures do not have the form of the GOE, and yet there is strong evidence that the Wigner surmise may still be applicable.

Brown and Langley tried to determine the conditions under which the natural frequency spacings of an engineering structure will have a Rayleigh distribution. To this end, numerical simulations have been performed for a simply supported flat plate, a simply supported string, and a system composed of two spring-coupled plates. In each case the structure was made uncertain by the addition of randomly placed masses or springs. It was found that the validity of the Wigner surmise depends on three factors: (i) the level of randomness, (ii) the degree of symmetry, and (iii) for the coupled system, on the strength of coupling between the two subsystems [23].

\section{RWD and normalization}

According to Wigner's surmise the statistical distribution of the widths and spacings of nuclear resonance levels is the Gaussian distribution $f_{\mathrm{G}}(x, t)$ multiplied by the independent variable $x$. This multiplication makes small distances less probable than those given by $f_{\mathrm{G}}(x, t)$. We put (for $x>0$ )

$$
f(x, t)=\frac{1}{2 D t} x \mathrm{e}^{-\frac{x^{2}}{4 D t}} .
$$

Then we notice that

$$
\frac{1}{2 D t} \int_{0}^{\infty} x \mathrm{e}^{-\frac{x^{2}}{4 D t}} \mathrm{~d} x=1
$$

and $f(x, t)$ is normalized.

\section{Diffusion equation corresponding to RWD}

For function (1) we find consecutively

$$
\begin{aligned}
& \frac{\partial f(x, t)}{\partial t}=\left(-\frac{1}{t}+\frac{x^{2}}{4 D t^{2}}\right) f(x, t), \\
& \frac{\partial f(x, t)}{\partial x}=\left(\frac{1}{x}-\frac{x}{2 D t}\right) f(x, t), \\
& \frac{\partial^{2} f(x, t)}{\partial x^{2}}=\left(-\frac{3}{2 D t}+\frac{4 x^{2}}{(4 D t)^{2}}\right) f(x, t),
\end{aligned}
$$

and

$$
D \frac{\partial^{2} f(x, t)}{\partial x^{2}}=\left(-\frac{3}{2 t}+\frac{x^{2}}{4 D t^{2}}\right) f(x, t) .
$$

Hence we observe that the function $f(x, t)$ satisfies the equation

$$
\frac{\partial f}{\partial t}=D \frac{\partial^{2} f}{\partial x^{2}}+\frac{1}{2 t} f
$$

in which the second terms on the right side is decreasing with the time $t$.

\section{Wigner's surmise counterpart on the time axis}

Another than (1) solution of Eq. (7) can be found. By substitution

$$
f(x, t)=\sqrt{t} u(x, t),
$$

we transform Eq. (7) into

$$
\frac{\partial u}{\partial t}=D \frac{\partial^{2} u}{\partial x^{2}}
$$

This is an ordinary equation of diffusion, which admits solution in the Gaussian form 


$$
u_{\mathrm{G}}(x, t)=\frac{1}{2 \sqrt{\pi D t}} \mathrm{e}^{-\frac{x^{2}}{4 D t}}
$$

and

$$
f(x, t)=\frac{1}{2 \sqrt{\pi D}} \mathrm{e}^{-\frac{x^{2}}{4 D t}} .
$$

Differently to the case of Wigner's distribution, when the Gaussian is multiplied by the spatial variable $x$, now the Gaussian is multiplied by the squared time variable $\sqrt{t}$. Comparing with Wigner's surmise (1) we see that in Eq. (10) the factor $x / t$ is lacking.

\section{Mean square displacement}

Consider the mean square displacement when stochastic distribution $f(x, t)$ is described by Eq. (7). We define

$$
\left\langle x^{2}\right\rangle=\int_{0}^{\infty} x^{2} f(x, t) \mathrm{d} x .
$$

Multiply both sides Eq. (7) by $x^{2}$ and integrate both sides with respect to $x$ from $x=0$ to $\infty$. We notice that integration by parts gives

$$
\int_{0}^{\infty} x^{2} \frac{\partial^{2} f}{\partial x^{2}} \mathrm{~d} x=2
$$

if we assume that at infinity both $f$ and $\partial f / \partial x$ vanish. We get

$$
\frac{\partial}{\partial t}\left\langle x^{2}\right\rangle=2 D+\frac{1}{2 t}\left\langle x^{2}\right\rangle
$$

which is satisfied if

$$
\left\langle x^{2}\right\rangle=4 D t \text {. }
$$

This is value twice greater than in classical one-dimensional diffusion or random walk problem, which permits to regard that the additional term $(1 / 2)\left\langle x^{2}\right\rangle$ at right hand side of Eq. (13) plays the role of the drift term known from Smoluchowski's equation [24, 25].

\section{Variance of Wigner's distribution}

In full agreement with (14) the variance of distribution $f$ given by (1) when found directly, is

$$
\sigma^{2}=\int_{0}^{\infty} x^{2} f \mathrm{~d} x=4 D t
$$

instead of $2 D t$ for the Gaussian distribution and the kurtosis

$$
\kappa=\left(\frac{1}{\sigma^{2}}\right)^{2} \int_{0}^{\infty} x^{4} f \mathrm{~d} x=\frac{32(D t)^{2}}{(4 D t)^{2}}=2
$$

is distinctly less than 3 , the value for the Gaussian, cf. $[26,27]$. The identity of results $(14)$ and (15) is an additional verification of Eq. (7).

\section{Conclusions}

1. Equation (7) was found whose special solution is Rayleigh-Wigner's distribution (1). Thus, it is possible to analyse the time evolutions of Rayleigh-Wigner's type distributions in an analogical manner as the Gaussian distributions are analysed by the diffusion (or heat) differential equations.
2. While most non-equilibrium systems have no analytical solutions for the distribution and correlation functions, in the case of RWD it is possible to give explicit formulae for the variance and kurtosis of the distribution. The variance of RWD is a linear function of time, as in Fick's classical diffusion, but is 2 times greater. The kurtosis has value 2 which is smaller than the value of classical diffusion equal 3 , and still is time independent.

3. It was observed that Rayleigh's distribution arises as a geometrical effect of the planar integration of random harmonic vibrations. Identity of Rayleigh's distribution and Wigner's surmise might indicate on an intrinsic relation of geometry of random harmonic motions and nuclear phenomena.

\section{Acknowledgments}

We are indebted to an unknown Referee for adverting our attention to Rayleigh's distribution, and criticism of my results in the first draft of the paper.

\section{References}

[1] J.W. Strutt, Lord Rayleigh, Philos. Mag. X, 73 (1880).

[2] J.W. Strutt, Lord Rayleigh, The Theory of Sound, Vol. 1, Sect. 42a, 2nd ed., Dover Publications, New York 1945

[3] E.P. Wigner, Math. Proc. Cambridge Philos. Soc. 47, 790 (1951).

[4] E.P. Wigner, SIAM Rev. 9, 1 (1967).

[5] H.L. Montgomery, Proc. Symp. Pure Math. Providence 24, 181 (1973).

[6] A.M. Odlyzko, Contemp. Math. 290, 139 (1999).

[7] N.M. Katz, P. Sarnak, Bull. AMS 36, 1 (1999).

[8] B. Hayes, Am. Scient. 91, 296 (2003).

[9] J. Derbyshire, Prime Obsession: Bernhard Riemann and the Greatest Unsolved Problem in Mathematics, Joseph Henry Press, Washington, DC 2003.

[10] J. Baik, A. Borodin, P. Deift, T. Suidan, J. Phys. A, Math. Gen. 39, 8965 (2006).

[11] J.S. Nicholson, Notices Am. Math. Soc. 59, 38 (2012).

[12] H.S. Carslaw, J.C. Jaeger, Conduction of Heat in Solids, Clarendon Press, Oxford 1948.

[13] L.D. Landau, Ya. Smorodinsky, Lectures on the Theory of Atomic Nucleus, Consultants Bureau, New York 1958

[14] M.L. Mehta, Nucl. Phys. 18, 395 (1960).

[15] M. Gaudin, Nucl. Phys. 25, 447 (1961).

[16] M. Krbálek, P. Šeba, Phys. Rev. E 64, 066119 (2001).

[17] M. Krbálek, P. Šeba, J. Phys. A, Math. Gen. 36, L7 (2003).

[18] M. Krbálek, P. Šeba, J. Phys. A, Math. Theor. 42, 345001 (2009).

[19] F. Michael, M.D. Johnson, Physica A 320, 525 (2003). 
[20] P. Šeba, Parking and the visual perception of space, arxiv/0907.1914v1 [physics.soc-ph], 10 Jul 2009.

[21] Centralna Komisja Egzaminacyjna, Fizyka i Astronomia, 2012, p. 105.

[22] Okregowa Komisja Egzaminacyjna, Analiza wyników egzaminów maturalnych z fizyki i astronomii, Raport 2012, p. 285.

[23] A.W.M. Brown, R.S. Langley, J. Acoust. Soc. Am. 109, 2443 (2001).
[24] S. Chandrasekhar, M. Kac, R. Smoluchowski, Marian Smoluchowski - his life and scientific work, Ed. R.S. Ingarden, PWN, Warszawa 1999.

[25] R.F. Streater, Rep. Math. Phys. 40, 557 (1997).

[26] R. Wojnar, Acta Phys. Pol. A 114, 607 (2008).

[27] R. Wojnar, Acta Phys. Pol. A 121, B-133 (2012). 